\title{
Hyperspectral Image Classification using Softcomputing Techniques: A Review
}

\author{
A. Rajitha \\ Dept. Computer Science \\ SPMVV, Tirupati-515502 \\ Andhra Pradesh, India
}

\author{
P. Bhargavi, PhD \\ Dept. Computer Science \\ SPMVV, Tirupati-515502 \\ Andhra Pradesh, India
}

\author{
S. Jyothi \\ Professor \\ Dept. Computer Science \\ SPMVV, Tirupati-515502 \\ Andhra Pradesh, India
}

\begin{abstract}
Hyperspectral image classification plays a major role in remote image analysis. Hyperspectral images provide both spatial details of airborne imagery and spectral resolution for spectroscopic analysis and narrow band analysis techniques. Available satellite sensors like Hyperion, HyMap and AVIRIS are good sources of hyperspectral data. Applications of hyperspectral images are remote sensing, seed viability study, biotechnology, environmental monitoring, medical diagnose, food, pharmaceuticals and so on. Traditional techniques are difficult to deal with hyperspectral images directly, because hyperspectral images have continuous narrow spectral bands. To overcome this, hyperspectral image classification can be done using different softcomputing techniques. Softcomputing is an emerging field consisting of Fuzzy Logic, Neural Network and Genetic Algorithms. This paper reviews how hyperspectral image classification can be done using different softcomputing techniques.
\end{abstract}

\section{Keywords}

Hyperspectral Image classification, Fuzzy Logic, Artificial Neural Networks, Genetic Algorithm

\section{INTRODUCTION}

Remote sensing can be defined as collection and interpretation of information about an object, area or event without any physical contact with object. Aircraft and satellites are the common platforms for remote sensing of earth and its natural resources [1]. Aerial photography in visible portion of the electromagnetic wavelength was the original form of remote sensing but technological developments has enabled the acquisition of information at other wavelength including near infrared, thermal infrared and microwave. Collection of information over a large numbers of wavelength bands is referred as hyperspectral data. Hyperspectral image analysis and interpretation concerns with spectra acquired from a given scene at a short, medium or long distance by an airborne or satellite sensor.

Hyperspectral Signature detects the individual absorption features of all materials, because all the materials are bound by chemical bonds. Hence hyperspectral data is used to detect fine changes in vegetation, soil, water and mineral reflectance. Hyperspectral remote sensing image analysis also attracts a growing interest in real-world applications such as urban planning, agriculture, forestry and monitoring. Hyperspectral data contain extremely rich spectral attributes, which offer the potential to discriminate more detailed classes with classification accuracy. Hyperspectral image classification is the process used to produce thematic maps from remote sensing image. A thematic map represents the earth surface objects (Soil, vegetation, roof, road, buildings) and its construction implies the themes or categories selected for the map are distinguishable in image [2].

Availability of advanced sensor technology such as NASA's Airborne Visible-Infrared Imaging Spectrometer (AVIRIS) has resulted in the collection of spectral data over terrestrial regions at high, medium and low altitudes through adjacent multi-band channels, enabling its utilization in a multitude of applications ranging from environmental planning and assessment, monitoring of oil spills, geological research, and target detection in military applications [3]. Hyperspectral imaging can be defined as the simultaneous acquisition of an image in many narrow contiguous spectral bands [4]. The advantage of this technique is that, considering that every element (water, tree, soil, etc) is defined by a spectrum (spectral signature).

The overall objective of image classification procedures is to automatically categorize all pixels in image into land cover classes [5]. Based on pixel information, Images can be classified as Per-pixel, Sub pixel, Per-field, Knowledge based, Contextual and multiple classifiers. Per-pixel classifiers may be parametric or non-parametric. Based on the use of training samples, images can be classified as Supervised and Unsupervised Classification. The unsupervised classification is the identification of natural groups or structures. The supervised classification is the process of using samples of known identity to classify (i.e.) to assign unclassified pixels to one of several informational classes.

Different softcomputing techniques can be used to classify the hyperspectral images. Softcomputing is tolerant of imprecision, uncertainty, partial truth, and approximation, unlike hardcomputing. In effect, the human mind is the role model for soft computing. Some of the important approaches in softcomptuing are Fuzzy Logic, Genetic Algorithm and Artificial Neural Networks [6].

With the concept and methods, applications of soft computing in the field of agricultural and biological engineering are presented especially in the soil and water context for crop management and decision support in precision agriculture [7]. This paper explains different softcomputing techniques on the domain of hyperspectral images such as Fuzzy Logic (FL), Artificial Neural Networks (ANN) and Genetic Algorithms (GA).

\section{HYPERSPECTRALIMAGE CLASSIFICATION}

Classification is a process in which individual items (objects) patterns/image regions/pixels) are grouped based on the similarity between the item and the description of the group. Hyperspectral image classification is to classify 
the image pixels within a hyperspectral image into multiple categories, which has received increasing research interests in a wide variety of applications [8-12]. The objective of image classification is to obtain the spectrum for each pixel in the hyperspectral image, with the purpose of finding objects, identifying materials, or detecting processes. Image classification is a process of sorting pixels in to individual classes, based on pixel values. This classification is used to assign corresponding levels with respect to groups and used as extraction techniques in digital remote sensing [13].

Hyperspectral image classification involves the identification of various pixels in the hyperspectral image sequence. This image sequence contains the spectral information. Each spectral sequence of hyperspectral images have unique electromagnetic spectrum, by using these electromagnetic spectrums each image distinguished with other hyperspectral images.

Classification of hyperspectral images is not the trivial task, as it requires so many factors to be concerned such as

- The large number of land-cover class to be handled.

- High number of spectral bands but low number of the availability of training samples. This phenomenon is known as Hughes Phenomenon [14] or 'curse of dimensionality'.

- Non-linear spread of the data classes.

To overcome these problems proper classifier should be selected. The classifier has to support the large data, multi class data and the nonlinear dataset. Significant features among the extracted features must be selected and to be fed to the classifiers to obtain the high accuracy rate. This process is commonly known as feature selection [15].

The hyperspectral classification falls into two major categories such as spectral classification and the spatial classification. In spectral classification, the reflectance values of the pixels at different wavelengths are considered. The pixels of different classes exhibit different reflectance at different wavelengths. From these wavelengths and reflectance, required spectral features such as mean reflectance, maximum reflectance, minimum reflectance, standard deviation, and variance can be calculated and can be used for classification. As this type of classification uses, spectral information it is known as spectral classification. In spatial classification, the spatial arrangement of pixels and their contextual values, textural properties are identified and features. Classification of hyperspectral image plays a vital role in different applications like, assessment of environmental damage, growth regulation, land use land cover mapping, urban planning, crop monitoring, forest applications etc,.

The major steps of image classification may include determination of a suitable classification system, selection of training samples, image pre-processing, and feature extraction, selection of suitable classification approaches, post-classification processing, and accuracy assessment. In general different hyperspectral image classification approaches are $[2,16]$.

- $\quad$ Based on pixel information, Images can be classified as Per-pixel, Sub pixel, Per-field, Knowledge based, Contextual and multiple classifiers. Per-pixel classifiers may be parametric or non-parametric.
- $\quad$ Based on the use of training samples, images can be classified as Supervised and Unsupervised Classification. The unsupervised classification is the identification of natural groups or structures. The supervised classification is the process of using samples of known identity to classify (i.e.) to assign unclassified pixels to one of several informational classes. Supervised method follows the steps such as feature extraction, training and labeling processes.

\subsection{Hyperspectral Image Classification Based on Pixel Information}

Based on pixel information, hyperspectral images can be classified as Per-Pixel, Sub Pixel, Per-field, Knowledge based, Contextual and multiple Classifiers.

\subsubsection{Per-pixel Classifier}

In Per-pixel Classifiers, each pixel is classified into only one category. For a given feature, Per-pixel classifiers are used to develop a signature by adding the spectra of all training set pixels. The resulting signature ignores the impact of mixed pixels and contains the contribution of every material present in the training pixels [5]. Per pixel classifiers may be parametric or non parametric. These methods can be used in decision trees and Support Vector Machine [17].

\subsubsection{Sub Pixel Classifiers}

Most classification approaches are based on per-pixel information in which each pixel is classified into one category and the land cover classes are mutually exclusive. Due to the heterogeneity of landscapes and the limitation in spatial resolution of remote sensing imagery, mixed pixels are common in medium and coarse spatial resolution data. Sub-pixel classification approaches have been developed to provide a more appropriate representation and accurate area estimation of land covers than per-pixel approaches especially when coarse spatial resolution data are used [18].

\subsubsection{Per- Field Classifiers}

Per-field classifier classifies land use by predetermined field boundaries, with an assumption that each field belongs to a single, homogeneous class [19]. Per-field classification is developed to overcome the weakness of per pixel classification. Per-field classification has the advantage of allowing incorporation of variety of field attributes such as size, shape, perimeter of the field as classification criteria. In Per-field classification, field boundaries are predetermined.

\subsubsection{Knowledge Based Classifiers}

Different kinds of ancillary data, such as digital elevation model, soil map, housing and temperature are readily available; they may be incorporated into a classification procedure in different ways. One approach is to develop knowledge based classifications based on the spatial distribution pattern of land cover classes and selected ancillary data [2]. Ref [20] was Summarized three methods employed to build rules for image classification. They are explicitly eliciting knowledge from experts, implicitly extracting variables and rules using cognitive methods and empirically generating rules from observed data with automatic induction methods. Ref. [21] was proposed a new scheme of knowledge based classification and rule generation using a fuzzy multilayer perceptron. 


\subsubsection{Contextual Classifiers}

In contextual classifiers, the spatially neighboring pixel information is used. Contextual classifiers are developed to cope with the problem of intraclass spectral variations [22]. To improve the classification results, it exploits spatial information among neighboring pixels [23]. It may use smoothing techniques, segmentation and neural networks.

\subsection{Hyperspectral Image Classification Based on Training Samples}

Training Samples are classified as Supervised Classification and Unsupervised Classification. In supervised classification, it identifies known a priori through a combination of fieldwork, map analysis as training sites; the spectral characteristics of these sites are used to train the classification algorithm for eventual land cover mapping of the remainder of the image. In Unsupervised Classification, the computer or algorithm automatically group pixels with similar spectral characteristics (means, standard deviations, etc.,) into unique clusters according to some statistically determined criteria [2].

\subsubsection{Supervised Classification}

In this type of classification the image analyst "supervises" the pixel categorization process by specifying the computer algorithm, numerical descriptors of the various land cover types present in a scene. Representative sample sites of known cover types, called training areas, are used to compile a numerical "interpretation key" that describes the spectral attributes of each feature type of interest. Each pixel in the data set is then compared numerically to each category in the interpretation key and labeled with the name of category it looks most similar.

Usually the analyst begins by assembling and studying maps and remote sensing images of the area to be classified and by investigating selected sites in the field. The objective is to identify a set of pixels that accurately represent spectral variation present within each informational region. The algorithm generates decision boundaries.

Various supervised classification methods may be used to assign an unknown pixel to one of a number of classes. The choice of a particular classifier or decision rule depends on the nature of the input data and the desired output. Among the most frequently used classification algorithms are the maximum likelihood, Bayesian, minimum distance and parallelepiped algorithms [16].

In supervised Classification, Land cover classes are defined. Sufficient reference data are available and used as training samples [5]. The signatures generated from the training samples are then used to train the classifier to classify the spectral data into a thematic map. Most frequently used supervised classification approaches are maximum likelihood, decision tree and neural network. One of the tasks carried out by Intelligent System is Supervised Classification. A large number of methods have been developed based on Perceptron based techniques (i.e.) Feed Forward Networks [24].

\subsubsection{Unsupervised Classification}

It can be defined as the identification of natural groups, or structures, within multispectral data. The notion of the existence of natural, inherent groupings of spectral values within a scene may not be intuitively obvious, but it can be demonstrated that remotely sensed images are usually composed of spectral classes that are reasonably uniform internally in respect to brightness in several spectral channels. The algorithm identifies clusters or groups of these similar data and the analyst identifies the individual clusters [16]

In Unsupervised Classification Clustering based algorithms are used to partition the spectral image into a number of spectral classes based on the statistical information inherent in the image. No prior definitions of the classes are used. The analysis is responsible for labeling and merging the spectral classes into meaningful classes. The unsupervised classification approaches are ISODATA and K-means Clustering Algorithm. One of the methods used in unsupervised classification technique is ISODATA [2,25] which uses a maximum- likelihood decision rule to calculate class. It can be evenly distributed in the data space and then iteratively clusters the remaining pixels using Minimum Distance techniques.

\section{SOFTCOMPUTING}

Softcomputing became a formal Computer Science area of study in early 1990s [26]. Softcomputing is a term used in computer science to refer the problem in computer science whose solution is not predictable, uncertain and between 0 and 1 . Soft computing is a relatively new concept, the term really entering general circulation in 1994. The term "soft computing" was introduced by Professor Lotfi Zadeh with the objective of exploiting the tolerance for imprecision, uncertainty and partial truth to achieve tractability, robustness, low solution cost and better rapport with reality. The ultimate goal is to emulate the human mind as closely as possible. Soft computing involves the different fields [27].

Soft computing is asset of "inexact" computing techniques, which are able to model and analyze very complex problems. For these complex problems, more conventional methods have not been able to produce cost effective, analytical, or complete solutions. Soft computing has been extensively applied in scientific research and engineering computing. In agriculture and biological engineering, soft computing techniques like, Fuzzy Logic, Artificial Neural Networks and Genetic algorithms are used to study soil and water regimes related to crop growth, analyzing the operation of food processing, and support decision making in precision farming.

Soft Computing is defined as a collection of techniques used in computational intelligence. Soft Computing has three main branches: Fuzzy Systems, Genetic Algorithms and Neural Networks.

\subsection{Fuzzy Logic}

Fuzzy Logic was introduced Lotfi A. Zadeh, Ph.D., University of California, Berkley. Fuzzy logic is an approach to computing based on 'degree of truth' rather than 'True or False'. The fuzzy systems convert these rules to their mathematical equivalents. The job of the system designer and the computer is simplified which results in accurate representations of the way systems behave in the real world. The value 0 and 1 describes 'not belonging to' and 'belonging to' a conventional set, respectively. While the values between 0 and 1 as described in [27] are represented as 'fuzziness'. Fuzzy sets [28] provide a robust mathematical framework for dealing with "real-world" 
imprecision and nonstatistical uncertainty. An important property of fuzzy set is that it allows partial membership. A fuzzy set is a set having the degrees of membership between 0 and 1 . The operations carried out on fuzzy set are all set operations such as Union, Intersection, Complement, etc. The set operations are shown by Venn diagram.

\subsubsection{Advantages}

- Simplicity and flexibility.

- It also allows vague linguistic terms in the rules.

\subsubsection{Disadvantage}

-It is difficult to identify membership function.

Fuzzy Logic makes conversion of imprecise information to precise one, consists of capability to design rational decisions containing imperfect information. Uncertainty, imprecision, incompleteness, risk management, partial true and vice versa is an attribute of information in Fuzzy systems [29]. Fuzzy controllers are simple, low cost and can be designed without knowing the mathematical model of the process. Fuzzy logic is one of the successful application of fuzzy set in which the variables are linguistic rather than the numeric variable. A fuzzy logic controller is based on a set of control the rules called as the fuzzy rules among the linguistic variables. These rules are expressed in the form of conditional statements. The fuzzy logic system contains three units fuzzification unit, decision making unit and defuzzification unit. The fuzzification unit converts the crisp value into a linguistic format. The decision unit decides in the linguistic format with the help of logical linguistic rules supplied by the rule base unit. The defuzzification unit takes the input from the decision making unit and convert the linguistic format of the signal into crisp form [30].

\subsection{Artificial Neural Network}

Artificial neural networks are used in pattern recognition and classification of remotely sensing images in recent years. Artificial neural networks (ANNs) are computational models inspired the brain, and are used to estimate or approximate functions that can depend on a large number of inputs and are generally unknown. Artificial neural networks are generally presented as systems of interconnected "neurons" which can compute values from inputs [31]

\subsubsection{Advantages}

- They can use it for non-linear problems.

- Using Back propagation learning algorithm is widely used in solving various classifications and forecasting problems.

\subsubsection{Disadvantages}

- It behaves as Black box system due to which the user cannot explain how learning from input data was performed.

Artificial neural networks (ANNs) are computer programs that are designed to simulate human learning process through establishment and reinforcement of linkages between input and output data. It is linkages that form the analogy with the human learning process in that repeated association between input and output in the training process reinforce linkages that can be employed to link impute and output in the absence of training data.
ANNs are composed of three elements. In input layer consists of the source, which in the contents of Remote Sensing is the multispectral observations, perhaps in several bands and from several dates. The output layer consists of the classes required by the analyst. Included are training data in which the association between output labels and input data are clearly established. During the trading phase, An ANNs establishes an association between input and output data by establishment of weights within one or more hidden layers. In context of Remote Sensing repeated association between classes and digital values, as expressed in the training data, strengthen weights want in hidden layers that permit the ANN to assign correct when given spectral values in the absence of training data, artificial neural networks have been employed to process multispectral remote sensing images and have achieved improved accuracy compared to those obtained traditional statically methods [32].

\subsection{Genetic Algorithms}

Genetic Algorithm is an optimization and heuristics search technique that uses techniques inspired by evolutionary biology search as fitness, mutation, selection and crossover. GA works simultaneously on set of potential solutions to the problem. Algorithm starts with a set of solutions called a sub population. the fitness to which solution meet some performance criterion is evaluated and used to select "surviving" individuals that will reproduce a new , better sub population. Then, individuals will conduct alterations similar to the natural genetic mutation and crossover. The selection scheme makes the process toward high performance solutions. A careful selection of genetic algorithm structures and parameters can ensure a good chance of reaching the globally optimal solutions after number of iterations.

Genetic Algorithm performs following operations [33].

- Fitness: It is used to evaluate Fitness score of an individual (feature). With the help of this score the selection process will be carried out

- Selection: It is used to select the chromosome from the population by using the fitness score.

- Crossover: Two individuals are chosen from the population using the selection operation. A position is selected in the chromosome. The bits lying after the selected position in the parent are swapped so as to produce new offspring.

- Mutation: Each new offspring is used and one bit or more than one bit will be flipped (1 becomes 0 and 0 becomes 1 ). It is used to maintain diversity within the population.

\subsubsection{Advantages}

- It can solve with multiple solutions.

- Every optimization problem which can be described with the chromosome encoding could be solved by it.

- It is easy to understand and it practically does not demand the knowledge of mathematics.

\subsubsection{Disadvantages}

- The population has lot of subjects which makes it difficult to the genetic algorithm to find a global optimum.

- It cannot assure constant optimization response 
Genetic Algorithms is alternative tools to traditional optimization methods. Genetic Algorithms have successfully used in many field such as scheduling, function optimization, mechanical learning an important method of softcomputing [34].

\section{SOFTCOMPUTING TECHNIQUES FOR HYPERSPECTRAL IMAGE CLASSIFICATION}

Different Remote sensing images are Multispectral and Hyperspectral images. Multispectral images refer 3-10 bands. Hyperspectral images consists of much narrows bands (10-200nm).Having a higher level of spectral detail in hyperspectral images gives better capability to see the unseen, identify and classify images more accurately. Soft computing techniques can be used efficiently for Remote sensing image classification. In conventional classification techniques, training and testing are done based on one pixel - one class method. Therefore conflictions are arising in classifying mixed pixels. Fuzzy Logic (FL) which takes into account of membership functions had been used for this purpose as an alternative method Zadeh proposed the concept of fuzzy logic in 1960s [35].

Genetic Algorithm (GA) which explains the natural evolution can be used for optimizing the fuzzy rules which in turn provides good classification result [36]. Artificial Neural Network (ANN) imitates the human brain, have a number of elements, similar to neurons which are interconnecting to do processing. Neural networks have to be trained to establish a relation between the input and output given during training. A good data set is required to draw better classification accuracy [37].

Naveen J.P. et al., [38] mentioned that soil fertility was estimated by using hyperspectral imaging. Papageriou and Chen et al.,[61,62] were design a fuzzy expert system to "Design and Development of Expert system for Potato Crop" analysis the soil condition using a fuzzy membership function. This decides to reduce the cost of fertile applied, grow the crops organically and protect the natural benefits of soil

Using hyperspectral images, Nedeljkovic et al., [39] classified SPOT image using fuzzy logic classification product. The results were compared with supervised classification. It was concluded that fuzzy logic takes advantages of already created simple rules and images classification in equal or less time consuming with satisfactory classification accurately.

Soil mapping using Fuzzy logic has been developed by Zhu et al. [40] and Shi et al. [41]. These two papers describe Soil land Inference Model (Slim) based on fuzzy soil inference system. This model consists three major components. i) A model employing a similarity representation of soils. ii) a set of inference techniques for deriving the similarity representation and iii) use of the similarity representation.

ANN research had experienced three periods of extensive activity. In 1940s, McCulloch and [42] proposed the first mathematical neuron model. This was followed by Rosenblatt's [43] perceptron theory in $1960 \mathrm{~s}$. Since the late $1980 \mathrm{~s}$, there has been a dramatic growth in the level of research activity in the neural network field. The major development was the introduction of back- propagation
The survey Regarding the large number of the data and considerable variations in soil layers in Sudan, was done by Elarabhi et al., [44]. In this Artificial Neural Network s is used to classify soils and predict the soil parameters. This study identifies positive results in soil classification with a high degree of success, especially when the distances between the boreholes are reasonable.

High resolution soil maps are generally available for small areas because obtaining these maps through fields survey is time consuming and expensive. Zhengyong Zhao et al [45], was developed an Artificial Neural Network (ANN) model to predict soil texture (sand, clay and slit contents) based on soil attributes. Here the ANN model is used to produce a high resolution soil maps in area with similar conditions without additional field surveys.

Applications of neural networks or neuro fuzzy model in crop models in crop mapping have been explored by many researches. Li R. et al., [46] demonstrated the ability of the feature-weighted detector (FWD) that enables the classification of pattern and selection features. Qui et al [47] developed a Gaussian Fuzzy Learning Vector Quantization (GFLVQ), which is supervised image classifier that makes use of proper knowledge in the training data to update a single neuron without complete retraining.

Verbeiren et al [48] studied potential of sub pixel classification for regional crop area estimation using time series of monthly NDVI- composites. Two different methods were investigated: the linear mixture model and neural networks. Both algorithms were trained with parts of the reference data and validated with the remainder. The best results are obtained with the NN estimates, for the most of the classes. The method is effective for wide scale, regional area estimation in data-poor countries. Wang, y. and Jamshidi. Et al., [49] proposed hierarchical Fuzzy Neural Network (HFNN) classifier in remote sensing data classification. In the HFNN classification, each FNN system is composed of FNN classifier and further FNN system need input for the output of previous FNN systems. In FNN classification without the hierarchical structure needed 1152 rules. However in HFNN classifier by combining more input bands, 40 rules is used totally with the same membership functions with improved accuracy. Wui. W et al., [50] presented a neuro fuzzy model to classify land use/land cover using Landsat 7ETM+ image. It combines neural networks and fuzzy systems to learn from the training data and generate conditional linguistic rules.

The backpropogation artificial neural network (ANN) is a well-known and widely applied mathematical model for remote sensing applications for pattern recognition, approximation and mapping of non linear functions and time-series prediction. Soo-See Chai et al., [51] described initial results on the best backpropogation ANN to be used to model the relationship between airborne microwave measurements of brightness temperature and soil moisture content. A number of different backpropogation ANN methods have been explored with the main criterion of analysis being the RMSE (Root Mean Square Error) between predicted soil moisture content and ground truth

In the study soil profiles, Odhiambo et al., [52] presented an application of a fuzzy neural network classifier for unsupervised clustering and classification of soil profile using ground-penetrating radar imagery. Freeland et at., 
[53] used two layer perception neural networks that perform supervised classification to examine the feasibility of using textural features extracted from ground penetrating radar for mapping subsurface soil conditions. Bajwa et al., [54] used multi layer feed forward ANN trained with GA to optimize the ANN topology and other four unsupervised and supervised methods to identify aerial hyperspectral image bands to characterize soil suitability in agricultural fields.

Fidencio et al., [55] applied CP ANN and RBF ANN in classification of soils using near-infrared spectroscopy. Altendorf et al., [56] developed a set of feed forward ANNs with BP training to predict soil water content at a given depth as a function of soil temperature. In the study of soil properties, Zhang et at., [57] applied ANNs to simulate the integration between soil and tillage and soil behavior.

In the study of soil temperature, Yang et al [58] developed a model based on feed forward ANN training with the BP algorithm to simulate daily soil temperatures at 100,500 and $1500 \mathrm{~mm}$ depth of soils.

Pachepsky et at., [59] developed stochastic imaging of the available soil water capacity(AWC) and estimates crop yield as related to soil sampling density and weather patterns. Parasuraman et al., [60] estimate saturated hydraulic conductivity using Genetic Programming (GP) and this is compared with the ANN model. Here GP appeared to be a promising tool for estimating the saturated hydraulic conductivity.

\section{CONCLUSION}

This review focused on hyperspectral image classification using softcomputing techniques. It summarizes different softcomputing techniques are used for hyperspectral image classification. Hyperspectral image classification is used in different areas like agriculture, medical diagnose, forensic and soon. The combination of different classification approaches have been shown to be helpful for improvement of classification accuracy.

\section{REFERENCES}

[1] A. F. H. Goetz, G. Vane, J. E. Solomon, and B. N Rock, "Imaging spectrometry for earth remote sensing," Science, vol. 228, no. 4704, pp. 1147-1153, Jun. 1985.

[2] R. Ablin, C. Helen Sulochana,"A Survey of Hyperspectral Image Classification", IJARCCE Vol.2, Issue 8, August 2013, 2319-5940.

[3] R.J. AspinallJ, W.J. Marcus, and A.W. Boardman "Considerations in collecting, processing, and analyzing high spatial resolution hyperspectral data for environmental investigations". Journal of Geographical Systems. 2002,4, 15-29.

[4] Richard J. Aspinall,W. Andrew Marcus, Joseph W. Boardman,"Considerations in collecting, processing, and analysing high spatial resolution hyperspectral data for environmental investigations", Journal of Geographical Systems, Volume 4, Issue 1, pp 15-29, March 2002.

[5] A. F. H. Goetz, G. Vane, J. E. Solomon, and B. N. Rock, "Imaging spectrometry for earth remote sensing," Science, vol. 228, no. 4704, pp. 1147-1153, Jun. 1985
[6] D. Lu \& Q. Weng. , "A Survey of Image Classification methods and techniques for improving classification Performance", International Journal of Remote Sensing, Vol 28, Issue 5, pp 823-870, 2007. [7] Swati Wakode, Rakesh Mallesh, Mandar Wagh , fManisha P Mali ," Classification of Unstructured Data using Soft Computing: A Survey", International Journal of Computer Science and Information Technologies, Vol. 6 , Issue 3, pp 2868-2870, 2015.

[8] B. Anil Gavade, S. Vijay Rajrobi "Productivity estimation and condition Assessment of horticulture crop from satellite based high resolution imaginary: A Review", User Interaction meet 2013, National Remote Sensing Center, Hyderabad, $21^{\text {st }}$ and $22^{\text {nd }}$ February 2013.

[9] D.Landgrebe, "Hyperspectral image data analysis", IEEE Signal Process Magazine, Vol. 19, Issue 1, pp. 17-28, 2002.

[10] B. Guo, S. Gunn, R. Damper, and J. Nelson, "Customizing kernel functions for svm-based hyperspectral image classification", IEEE Transaction on Image Processing, Vol. 17, Issue 4, pp. 622-629, 2008.

[11] H. Jiao, Y. Zhong, L. Zhang, and P. Li, "Unsupervised remote sensing image classification using an artificial dna computing," International Conference on Computing, Networking and Communications, 2011.

[12] O. Eches, N. Dobigeon, C. Mailhes, and J. Tourneret, "Bayesian estimation of linear mixtures using the normal compositional model. application to hyperspectral imagery," IEEE Transaction on Image Processing, vol. 19, no. 6, pp. 1403-1413, 2010.

[13] T. Liu, L. Zhang, P. Li, and H. Lin, "Remotely sensed image retrieval based on region-level semantic mining," EURASIP J. Image and Video Processing, vol. 4, 2012.

[14] T. Sheath, G. Nagalaxmi, and S. Jyothi, "A Study on Hyperspectral Remote Sensing Classification", International Journal of Computer Applications (0975 - 8887) International Conference on Information and Communication Technologies (ICICT- 2014).

[15] G. F. Hughes, "On the mean accuracy of statistical pattern recognizers, "IEEE Trans. Inf. Theory, vol. IT-14, no. 1, pp. 55-63, Jan. 1968.

[16] K. Kavitha and. S. Arivashagan, "A Novel Feature Derivation Technique for SVM based Hyper Spectral Image Classification", (C2010 International Journal of Computer Applications (0975 - 8887) Volume 1 No. 15.

[17] Sneha Murmua ,Sujata Biswas, "Application of Fuzzy logic and Neural Network in Crop Classification: A Review", International Conference On Water Resources, Coastal And Ocean Engineering (Icwrcoe 2015)

[18] Friedl, M.A, Brodley C.E, and Strahler A.H ,(1999), Maximizing land cover classification accuracies produced by decision trees at continental to global scales, IEEE Transactions on Geoscience and Remote Sensing, 37,969-977. 
[19] Foody G.M, Cox D.P., (1994), Sub pixel landcover composition estimation using a linear mixture model and fuzzy membership functions, Int. Remote sensing, 15, 619- 631

[20] Aplin,P. Atkinson ,M.P,Curran,J.P., (1999), Fine spatial resolution simulated satellite sensor imagery for land cover mapping in the united kingdom, Remote sensing of environment, 66, 206- 216.

[21]. Hodgson. M.E , John R.Jensen, Jason A.Tullis, Kevin .D.Riordan and Clerk M.Archer, (2003), Synergistic use Lidar and color Arial photography for mapping urban parcel imperviousness, Photogrammetric Engineering and remote sensing, $69,973-980$

[22]. Mitra,S. Rajat.K.De and Pal,S.K (1997),KnowledgeBased Fuzzy MLP for Classification and Rule Generation, IEEE Transactions on neural networks, $8,1338-1350$

[23]. Gong.P and Howaeth P.J, (1992), Frequencybased contextual classification and gray level vector reduction for land use identification, Photogrammetric Engineering and Remote sensing,,58,423-437.

[24] Magnussen.S, Boudewyn. P and Wulder.M, (2004), Contextual Classification of Landsat TM images to forest inventory cover types. International Journal of Remote Sensing. 24 2421-2440

[25] Kotsiantis.S.B, (2007), Supervised Machine Learning: A Review of Classification Techniques, Informatica 3, 249-268.

[26] Melesse .M. A and Jordan,J.D, (2002), Photogrammetric Engineering and Remote Sensing, 68.

[27] Zadeh, Lotfi A., "Fuzzy Logic, Neural Networks, and Soft Computing", Communication of the ACM, vol. 37, no. 3, pp. 77-84, 1994.

[28] "Principles of Soft Computing”, by ,Dr. S. N. Sivanandam, Dr. S. N. Deepa, $2^{\text {nd }}$ Edition.

[29] Sumit Ghosh, Qutaiba Razouqi,H. Jerry Schumacher, and Aivars Celmins "A Survey of Recent Advances in Fuzzy Logic in Telecommunications Networks and New Challenges"IEEE transactions on fuzzy systems, vol. 6, no. 3, August 1998 (443-447)

[30] Kailan Shay, Zakur Hussain, Applying Fuzzy :ogic to Risk Assessment and Decision Making, Nonember2013.

[31] Miss Maya, V. Mawale, Dr. Vinay Charan "Implementation and Simulation of Fuzzy Logic Controllers for productivity and fertility of soils and performance evaluation of Triangular Membership functions", COMPUSOFT, An International joining of advanced Computer Technology, 3(a), September2014.

http://en.wikipedia.org/wiki/Artificial_neural_networ $\mathrm{k}$.

[32] Sneha Murma, Sujatha Bizwas, "Applications of Fuzzy Logic and Neural Networks in crop classification: Review", International Conference On
Water Resources, Coastal And Ocean Engineering (Icwrcoe 2015), Aquatic Procedia 4(2015), 12041210.

[33] Swati Wakode, Rakesh Mallesh 1, Mandar Wagh 1 , Manisha P Mali , "Classification of Unstructured Data using Soft Computing: A Survey" (IJCSIT) International Journal of Computer Science and Information Technologies, Vol. 6 (3) , 2015, 28682870

[34] Yanbo Huagng, YUbn Lan, Steven J. Thomson Alex Fang, Wesley C, Ronald E Lacey, "Development of Soft Computing and applications in agricultural and biological engineering, Computers and Electronics in Agriculture, 7/2010 107-127.

[35] F. Wang, Geosci. Remote Sens. IEEE Trans. 28 (2) (1990) 194-201

[36] Z. Wang , X. Sun , D. Zhang , in: Advanced intelligent computing theories and applications. With aspects of artificial intelligence, 2007,pp.377384 .

[37] G.S. Dwarakish, B. Nithyapriya "Application of soft computing techniques in coastal study -A review," Journal of Ocean Engineering and Science 1 (2016) 247-255.

[38] Naveen J. P. Anne et. At. "Modeling Soil parameters using hyperspectral image reflectance in subtopical coastal wetlands", International journal of Applied Earth observation and Geoinfomatics" , 2014, pp 47-56

[39] Papageorgiou, Elpiniki I., Athanasis T, Markinos, and T. A. Gemtos. "Fuzzy cognitive map based approach for predicting yield in cotton crop production on a basis for decision support system in precision agriculture application" Applied Soft Computing 11.4(2011): 3643-3657.

[40] Zhu A Hudson, B. Burt, J. Lubich, “soil mapping using GIS ,expert knowledge and fuzzy logic ", soil science, Sociecty of American Journal, $65,1463-1472$.

[41] Shi X, Zhu A, Burt J, F Simsoon, "A case based necessary approach to fuzzy system mapping", 68 , 885-894.

[42] W.McCulloch , W. Pitts , Bull. Math. Biophys. 5 (1943) 115-133

[43] F. Rosenblatt, Psychol. Rev. 65 (1958) 386-408 .

[44] H. Elarabi, K. Ali ," Soil classification modeling using Artificial Neural Network", International Conference on Intelligent System, Dec 2008.

[45] Zhengyong Zhao, Thien Lien Chow, Herb W. Rees, Qi Yang, Zisheng Xing, Fan-Rui Meng, "Predict soil texture distributions using an artificial neural network model", Computers and Electronics in Agriculture 65(2009) 36-48

[46] Li R., Mukaidono, M., Burhan Turksen, I., 2002. A fuzzy neural network for pattern classification and feature selection. Fuzzy Sets and Systems 130, 101 108. 
[47] Qiu, F., 2008. Neuro-fuzzy Based Analysis of Hyperspectral Imagery. Photogrammetric Engineering \& Remote Sensing 74(10), 1235-1247.

[48] Verbeiren, S., Eerens, H., Piccard, I., Bauwens, I., V. Orshoven, J., 2008. Sub-pixel classification of SPOT-VEGETATION time series for the assessment of regional crop areas in Belgium. International Journal of Applied Earth Observation and Geoinformation, International Journal of Applied Earth Observation and Geoinformation 10(4), 486497.

[49] Wang, Y., Jamshidi, M.,2007. A Hierarchical Fuzzy Classification Scheme for Remote Sensing Data. Intelligent Automation and Soft Computing 13(4), 463-476.

[50] Wei, W., Guanglai, G., 2008. An application of neuro-fuzzy system in remote sensing image classification. International Conference on Computer Science and Software Engineering, IEEE, 1069-1072.

[51] Soo-See Chai, Bert Veenendaal, Geoff West, Jeffrey Philip Walker, "Backpropagation neural networks for soil moisture retrieval using NAFE'05 data:

[52] Odhiambo, L.O., Yoder, R.E., Yoder, D.C., 2001a. Estimation of reference crop evapo transpiration using fuzzy state models. Transactions of the ASAE 44(3), 543-550.

[53] Freeland, R.S., Odhiambo, L.O., 2007. Subsurface characterization using textural features extracted from GPR data. Transactions of the ASABE 50 (1), 287 293.

[54] Bajwa, S.G., Bajcsy, P., Groves, P., Tian, L.F., 2004. Hyperspectral image data mining for band selection in agricultural applications. Transactions of the ASAE 47 (3), 895-907.

[55] Fidencio, P.H., Ruisanchez, I., Poppi, R.J., 2001. Application of artificial neural networks to the classification of soils from Sao Paulo state using nearinfrared spectroscopy. Analyst 126, 2194-2200.

[56] Altendorf, C.T., Elliott, R.L., Stevens, E.W., Stone, M.L., 1999. Development and validation of a neural network model for soil water content prediction with comparison to regression techniques. Transactions of the ASAE 42 (3), 691-699.

[57] Zhang, Z.X., Kushwaha, R.L., 1999. Application of neural networks to simulate soil-tool interaction and soil behavior. Canadian Agricultural Engineering 41 (2), 119-125.

[58] Yang, C.C., Prasher, S.O., Lacroid, R., Kim, S.H., 2004b. Application of multivariate adaptive regression splines (MARS) to simulate soil temperature. Transactions of the ASAE 47 (3), 881887.

[59] Pachepsky, Y., Acock, B., 1998. Stochastic imaging of soil parameters to assess variability and uncertainty of crop yield estimates. Geoderma 85 (2-3), 213-229.

[60] Parasuraman, K., Elshorbagy, A., Si, B.C., 2007. Estimating saturated hydraulic conductivity using genetic programming. Soil Science Society of America Journal 71, 1676-1684.

[61] Papageorgiou, Elpiniki I., Athanasios T. Markinos, and T. A. Gemtos. "Fuzzy cognitive map based approach for predicting yield in cotton crop production as a basis for decision support system in precision agriculture application." Applied Soft Computing 11.4 (2011): 3643- 3657.

[62] Chen, Yun, Shahbaz Khan, and Zahra Paydar. "To retire or expand? A fuzzy GIS-based spatial multicriteria evaluation framework for irrigated agriculture." Irrigation and drainage 59.2 (2010): 174188 . 